\title{
Concentrated Beet Crystals
}

National Cancer Institute

\section{Source}

National Cancer Institute. Concentrated Beet Crystals. NCI Thesaurus. Code C114291.

A formulation containing concentrated beetroot crystals, with potential antioxidant and protective activities. Concentrated beetroot crystals contain antioxidants, including betacyanin, which scavenge free radicals, and high levels of nitrates and folic acid. Intake of concentrated beetroot crystals leads to the conversion of nitrate to nitric oxide (NO). This may have a beneficial effect on blood flow and blood pressure through the stimulation of NO-mediated vasodilation. Additionally, this agent may decrease fatigue and increase physical performance. 